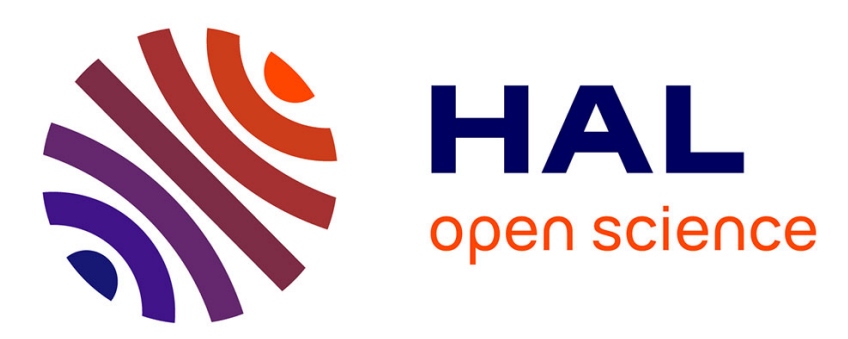

\title{
A FFT-based method to compute the permeability induced by a Stokes slip flow through a porous medium
}

\author{
Vincent Monchiet, Guy Bonnet, Guy Lauriat
}

\section{To cite this version:}

Vincent Monchiet, Guy Bonnet, Guy Lauriat. A FFT-based method to compute the permeability induced by a Stokes slip flow through a porous medium. Comptes Rendus Mécanique, 2009, 337 (4), pp.192-197. 10.1016/j.crme.2009.04.003 . hal-00687819

\section{HAL Id: hal-00687819 https://hal.science/hal-00687819}

Submitted on 18 Apr 2012

HAL is a multi-disciplinary open access archive for the deposit and dissemination of scientific research documents, whether they are published or not. The documents may come from teaching and research institutions in France or abroad, or from public or private research centers.
L'archive ouverte pluridisciplinaire HAL, est destinée au dépôt et à la diffusion de documents scientifiques de niveau recherche, publiés ou non, émanant des établissements d'enseignement et de recherche français ou étrangers, des laboratoires publics ou privés. 


\author{
A FFT-based method to compute the \\ permeability induced by a Stokes slip flow \\ through a porous medium \\ Vincent Monchiet, Guy Bonnet, Guy Lauriat \\ Université Paris-Est, Laboratoire Modélisation et Simulation \\ Multi Echelle, \\ LMSME FRE3160 CNRS, \\ 5 boulevard Descartes, 77454 Marne la Vallée, France
}

\title{
Résumé
}

The paper presents a FFT based-method for obtaining the permeability of a periodic micro-porous medium. The periodic medium is constituted of a rigid solid matrix saturated by a viscous fluid. The flow obeys the Stokes equations and a slip condition at the surface of the rigid skeleton is considered. The permeability is obtained from the homogenization of periodic media and an extension of the FFT method used for composite elastic media. The incorporation of the slip condition is made trough the introduction of an interphase between the fluid and the solid. The method of solution uses the classical expansion along Neumann series of the velocity field of the periodic problem and Green's tensor in Fourier space.

To cite this article :

\section{Résumé}

Cet article présente une méthode basée sur la FFT pour la détermination de la perméabilité d'un milieu poreux périodique. Le milieu poreux est constitué d'une matrice solide indéformable saturée par un fluide visqueux. L'écoulement obéit aux équations de Stokes et une condition de glissement sur la surface du squelette solide est également considérée. La perméabilité est alors obtenue en résolvant les équations issues de l'homogénéisation de milieux périodiques et par une extension de la méthode FFT utilisée pour les matériaux composites élastiques périodiques. La prise en compte de la condition de glissement est effectuée en introduisant une interphase entre le fluide visqueux et le solide. La méthode repose sur une représentation du champ de vitesses en séries de Fourier et sur l'expression explicite du tenseur de Green dans l'espace de Fourier. Pour citer cet article :

Key words: Porous media, homogenization, effective properties, permeability, viscous fluid

Mots-clés : Milieux poreux, homogénéisation, propriétés effectives, perméabilité, fluide visqueux 


\section{Introduction}

The determination of the effective properties of porous media, and particularly the permeability, is of fundamental importance in several practical problems in geomechanics, biomechanics, among others, and it has been the subject of many studies. Fluid flow through porous media is usually modeled using a volume-averaged approach and Darcy's law is valid for fluid flows with pore Reynolds numbers less than one. For forced flows, Darcy's law reads:

$$
Q_{i}=-\frac{k_{i j}}{\mu} \nabla_{j} P
$$

where $Q_{i}$ and $\nabla_{i} P$ are respectively the components of the flux and of the pressure gradient. $\mu$ is the dynamic viscosity and $k_{i j}$ the components of the permeability tensor of the considered medium. The determination of the permeability of a periodic porous medium knowing the geometry of the microstructure has been studied by many authors, see for instance [1], [2], [3], [4]. The methods to obtain a solution of the problem at the microscopic scale include expansion into series of eigenfunctions, finite element methods, etc. However, in many instances, as in the case of rarefied gases when the Knudsen number is small, a slip may occur at the surface of the skeleton. A semi-numerical method for the determination of the permeability in the case of a slip of flow the velocity field has been proposed by [5] but such a method is limited to specific inclusions. The aim of the present paper is to propose a FFT-based method to compute the permeability of a periodic porous medium including the slip effect. The Fourier based-method has been firstly introduced by Moulinec and Suquet [6] for the determination of the effective properties of elastic composites on the basis of previous works $[7,8]$. However the case of infinite contrast such as the case of rigid inclusions cannot be handled easily by the first method and so, the authors have proposed an alternate scheme based on augmented Lagrangian method [9]. The case of rigid inclusions can also be solved by using the dual formulation [10] which is extended to the case of porous media in the present paper. The consideration of a slip condition introduces a discontinuity in the velocity field at the surface of the rigid inclusions. Following [11], this discontinuity is introduced by considering an interphase of lower viscosity, between the fluid and the rigid inclusions. In fine, it is shown that the obtained iterative scheme takes a form similar to the one used for linear composites. The differences lie in the consideration of the first term introduced in this iterative scheme and in the recovering of the velocity field in our case, while elasticity problems need only deformation and stress fields. The methodology

Email addresses: vincent.monchiet@univ-Paris-est.fr (Vincent Monchiet), guy.bonnet@univ-Paris-est.fr (Guy Bonnet), guy.lauriat@univ-Paris-est.fr (Guy Lauriat). 
used to build this first term is depicted in section 4 and consists in introducing body forces in the rigid inclusion. An application to the case of aligned rigid cylinders is proposed in the last part of the paper.

\section{Statement of the problem}

Following $[12,13,14,15]$ and $[16,17]$ for slip flow, we consider a periodic medium made up of a rigid skeleton and of an incompressible viscous fluid obeying the Stokes equations with a slip condition:

$$
\left\{\begin{array}{l}
\mu_{f} \Delta \underline{v}(x)+\nabla p(x)=0, \quad \operatorname{div} \underline{v}^{*}(x)=0, \quad \forall \underline{x} \in V_{f} \\
v_{n}^{*}(\underline{x})=0, \quad \underline{v}_{t}^{*}(\underline{x})=c \nabla_{n} \underline{v}_{t}(\underline{x}) \quad \forall \underline{x} \in \Gamma
\end{array}\right.
$$

where $c$ is the "slip coefficient" function of the nature of the fluid and the solid, $\underline{v}^{*}(x)$ and $p(x)$ are the local velocity field and pressure, respectively. $\Gamma$ defines the surface of the solid and $\underline{n}$ is the normal unit vector taken on the surface $\Gamma$. I8n (2), $V_{f}$ is volume of occupied by the fluid. By $v_{n}^{*}(\underline{x})$ and $v_{t}^{*}(\underline{x})$, we denote the normal and tangential components of the velocity field $\underline{v}^{*}(\underline{x})$ respectively. By $\nabla_{n}$, we denote the normal gradient operator defined by $\nabla_{n}=\underline{\nabla} . \underline{n}$. The flow of the fluid is generated by a prescribed macroscopic pressure gradient, denoted by $G=\nabla P$. The local pressure reads $p(x)=G_{k} x_{k}+p^{*}(x), p^{*}(x)$

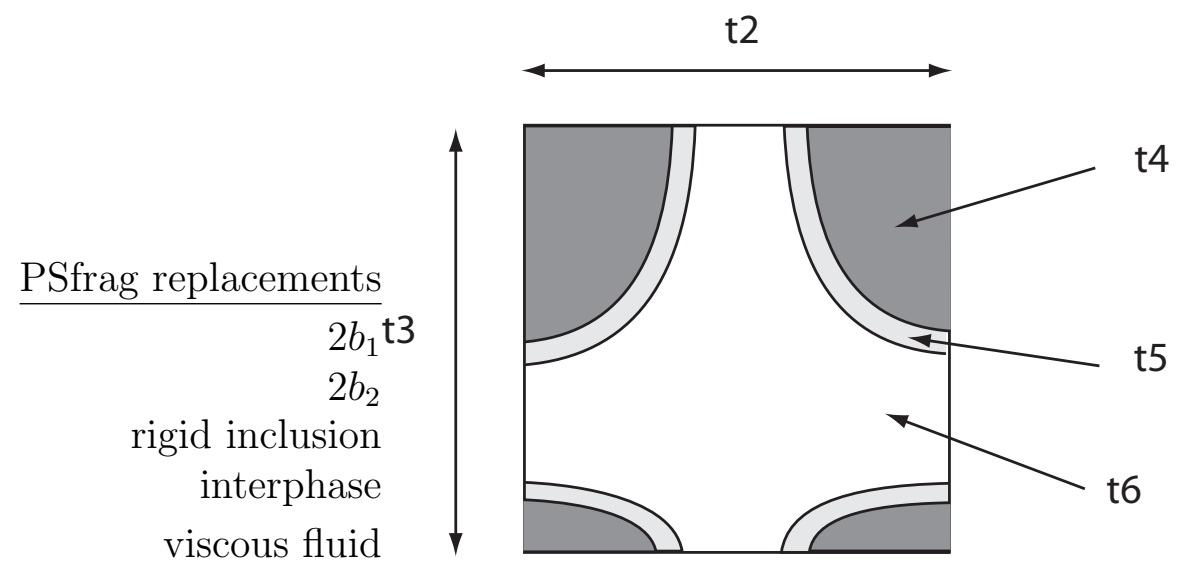

Figure 1. Periodic unit cell of the porous medium.

being periodic. At the local scale, the components of the velocity field $v_{i}^{*}(x)$ linearly depends on the components of the macroscopic pressure gradient :

$$
v_{i}^{*}(x)=A_{i j}(x) G_{j}
$$


where $A_{i j}(x)$ depends on the position vector, $x$. As shown in the framework of the homogenization applied to porous media [13], the average value over the unit cell of the velocity field reads:

$$
<v_{i}^{*}(x)>_{V}=\frac{1}{V} \int_{V} v_{i}^{*}(x) d V=<A_{i j}(x)>_{V} G_{j}=-\frac{k_{i j}}{\mu} G_{j}
$$

where $k_{i j}$ are the components of the permeability tensor. So, the computation of the permeability requires to determine the local velocity $\underline{v}^{*}(x)$. That can be done by using a FFT based-method. However, the slip condition implies to introduce a discontinuity in the velocity field at the surface $\Gamma$ of the inclusion. To take into account the slip condition, we introduce an interphase located between the rigid inclusions and the viscous fluid (see figure 1). This interphase is defined by a layer which has a constant thickness denoted $e$. The dynamic viscosity of this layer is denoted $\mu_{e}$. The local problem (2) is replaced by :

$$
\mu(x) \Delta \underline{v}^{*}(x)+\nabla p(x)=0, \quad \operatorname{div} \underline{v}^{*}(x)=0, \quad \underline{v}^{*}(x)=0 \quad \forall x \in \Gamma
$$

where the velocity field is null on the the surface $\Gamma, \mu(x)=\mu_{f}$ in the fluid and $\mu(x)=\mu_{e}$ in the interphase. The dynamic viscosity $\mu_{e}$ is chosen on the form $\mu_{e}=\left(e \mu_{f}\right) / c$ which allows to introduce a slip on the form given by $(2)$ when $e / a \rightarrow 0, a$ being a representative lengthscale of the inclusions (see [11] for more details about the method).

\section{The stress formulation of the problem and its solution}

In this section, a "stress based" formulation of the problem is depicted. Its solution is given in the Fourier space. We suppose first that the interphase has a finite thickness and the limited case, $e \rightarrow 0$ is examined secondly.

In the fluid, the local stress can be put into the form $\sigma=\Sigma+\sigma^{*}$ where $\sigma_{i j}^{*}$ is a periodic stress field and $\Sigma_{i j}$ is given in terms of the macroscopic pressure gradient, $\Sigma_{i j}=G_{k} x_{k} \delta_{i j}$. In the rigid solid, the stress is undefined. However, we propose to make a continuation by continuity of $\sigma^{*}$ and $\Sigma$ within the solid. We also introduce:

$$
\mathrm{M}(x)=\left\{\begin{array}{ll}
\mathrm{M}_{f} & \text { in the fluid } \\
\mathrm{M}_{s}=0 & \text { in the solid } \\
\mathrm{M}_{e} & \text { in the interphase }
\end{array} \quad \text { with: } \mathrm{M}_{\alpha}=\frac{1}{2 \mu_{\alpha}} \mathrm{K} \quad(\alpha=f, s,(\varnothing))\right.
$$


where $\mu_{s} \rightarrow+\infty$ within the solid, $\mathrm{K}=\mathrm{I}-\mathrm{J}$ where $\mathrm{I}$ is the fourth order identity tensor and $\mathrm{J}_{i j k l}=\delta_{i j} \delta_{k l} / 3$. The local problem (5) can thus be cast under the following form:

$$
\operatorname{div}\left(\sigma^{*}\right)+\underline{f}(x)=0, \quad \varepsilon^{*}(x)=\mathrm{M}(x): \sigma^{*}(x), \quad \varepsilon^{*}(x)=\operatorname{grad}_{s}\left(\underline{v}^{*}\right)
$$

where $\varepsilon^{*}$ are the components of the strain rate and $f_{i}=\Sigma_{i j, j}$. Introducing a reference medium defined by the dynamic viscosity $\mu_{0}$ and the fourth-order tensor $\mathrm{M}^{0}=\mathrm{K} /\left(2 \mu_{0}\right)$ (see [10] for more details) it is easy to show that the periodic stress field complies with :

$$
\sigma^{*}(\xi)=-\Omega(\xi) \cdot f(\xi)-\Delta^{0}(\xi):\left[\varepsilon^{*}(\xi)-\mathrm{M}^{0}: \sigma^{*}(\xi)\right]
$$

where $\Delta^{0}(\xi)$ is the periodic fourth-order Green's operator for the stress associated to the reference medium $\mathrm{M}^{0} . \Omega(\xi)$ is a third order tensor whose components are given by:

$$
\Omega_{i j k}(\xi)=\frac{i}{|\xi|^{4}}\left[\left(\delta_{i j} \xi_{k}+\delta_{i k} \xi_{j}+\delta_{j k} \xi_{i}\right)|\xi|^{2}-2 \xi_{i} \xi_{j} \xi_{k}\right]
$$

where $|\xi|$ denotes the norm of the wave vector $|\xi|=\left(\xi_{i} \xi_{i}\right)^{1 / 2}$. The periodic strain field, $\varepsilon^{*}(\xi)$, is defined by:

$$
\varepsilon^{*}(\xi)=\sum_{\alpha} \frac{1}{2 \mu_{\alpha}}\left[I_{\alpha}(\xi) * \sigma^{*}(\xi)\right] \quad \text { with }: I_{\alpha}(\xi)=\frac{1}{V} \int_{V_{\alpha}} \exp (i \xi . x) d x
$$

In the last relation $I_{\alpha}(\xi) * \sigma^{*}(\xi)$ denotes the convolution product between $I_{\alpha}(\xi)$ and $\sigma^{*}(\xi)$. The slip condition at the surface $\Gamma$ is accounted for by taking the limit $e / a \rightarrow 0$. It follows that:

$$
\begin{array}{r}
\varepsilon^{*}(\xi)=\frac{1}{2 \mu_{f}}\left[I_{f}(\xi) * \sigma^{*}(\xi)+\gamma I_{g}(\xi) * \sigma^{*}(\xi)\right] \\
\text { with: } I_{g}(\xi)=\frac{a}{V} \int_{\Gamma} \exp (i \xi \cdot x) d x
\end{array}
$$

in which we have introduced $\gamma=c / a$. Note that, in (8), the main difference with the case of elastic composites lies in the presence of : (i) the body force $f_{i}$ whose main consequence on the iterative scheme is explained in the following section, (ii) the slip condition introduced by $I_{g}(\xi)$. 


\section{The FFT based iterative scheme}

Relation (8) is the Fourier transform of an integral equation of "LippmanSchwinger-Dyson" type which can be obtained by the use of the equivalent explicit recurrence process:

$$
\sigma^{* i+1}(\xi)=-\Omega(\xi) \cdot f(\xi)-\Delta^{0}(\xi):\left[\varepsilon^{* i}(\xi)-\mathrm{M}^{0}: \sigma^{* i}(\xi)\right]
$$

starting from a first term $\sigma^{* 1}$ which will be defined later. Using the following equality:

$$
\Delta^{0}(\xi): \mathrm{M}^{0}: \sigma^{* i}(\xi)=\sigma^{* i}(\xi)+\Omega(\xi) \cdot f(\xi)
$$

the recurrence relation (13) can also be written in the form:

$$
\sigma^{* i+1}(\xi)=\sigma^{* i}(\xi)-\Delta^{0}(\xi): \varepsilon^{* i}(\xi)
$$

in which the "body forces", $f_{i}$, do not appear. The iterative scheme (15) takes the same form as in the case of an elastic composite. However, the main difference lies in the choice of $\sigma^{* i}$ at the first step in (15). This stress tensor must be periodic and must equilibrate the macroscopic gradient of pressure, of components $G_{i}$, within the fluid. It is not therefore divergence free as for elasticity. However, the associated strain rate field may not comply with the compatibility equation. Such a field is chosen, in the Fourier space, as $\sigma^{* 1}(\xi)=-\Omega(\xi) . f(\xi)$ where $f_{i}=G_{i}$ within the fluid but $f_{i}$ remains undefined within the inclusion. If we consider a uniform "body force" $f_{i}$ within the inclusion and considering the equilibrium of the unit cell, $\left\langle f_{i}\right\rangle_{V}=0$, it is easy to check that $f_{i}$ is given by:

$$
\begin{gathered}
f_{i}(x)= \begin{cases}G_{i} & \text { for } x \in V_{f} \\
-\frac{1-c_{s}}{c_{s}} G_{i} & \text { for } x \in V_{s}\end{cases} \\
\text { or: } f_{i}(\xi)= \begin{cases}-\frac{G_{i}}{c_{s}} I_{s}(\xi) & \xi_{i} \neq 0 \\
0 & \xi_{i}=0\end{cases}
\end{gathered}
$$

where $c_{s}$ defines the volume fraction of inclusions. Note that the "body force" within the inclusion is necessary in order to fix the inclusion. Note also that there are other possibilities to fix the inclusion, by considering a line force for example. The local strain is known at convergence of the iterative process and then, one can compute the components of the local velocity field as follows: 


$$
v_{i}^{*}(\xi)=\frac{2 i}{|\xi|^{2}} \varepsilon_{i j}^{*}(\xi) \xi_{j}
$$

At this stage, the velocity field is defined by its Fourier coefficients for all values of $\xi_{i}$ except for $\xi_{i}=0$. It means that the velocity field is defined up to an added constant. The reason for such a result is that the condition that $v_{i}^{*}$ is null at the surface of the solid has never been taken into account. The velocity field has to be computed from the one given by (18) but one has to specify that $v_{i}^{*}=0$ at one point taken within the rigid inclusion.

\section{Application}

As an application, we consider a periodic porous medium made up of rigid cylinders (of radius $a$ ) aligned in the plane of their cross sections and arranged along a squared lattice of area $4 b^{2}$. In the case of a rigid cylinder located at
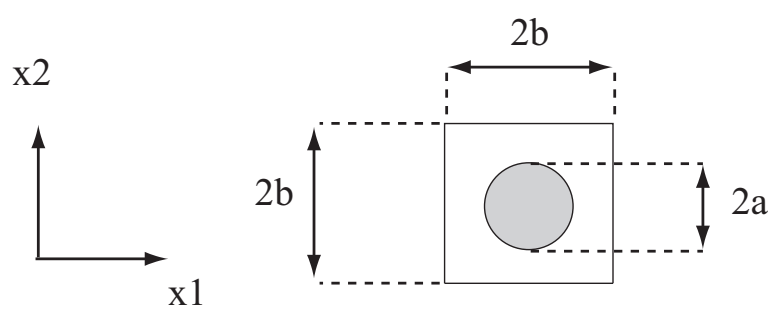

Figure 2. The unit cell for periodic array of aligned rigid cylinders

$x_{i}=0$, the shape coefficients $I_{s}(\xi)$ and $I_{g}(\xi)$ are given by:

$$
I_{s}(\xi)=\frac{\pi a}{2 b^{2}|\xi|} J_{1}(a|\xi|), \quad I_{g}(\xi)=\frac{\pi a^{2}}{2 b^{2}} J_{0}(a|\xi|)
$$

where $J_{0}$ and $J_{1}$ are the Bessel functions of the first kind, and zero and first order, respectively. The flow of the incompressible fluid is due to a prescribed macroscopic pressure $G_{i}=G_{1} \delta_{i 1}$. Due to the squared symmetry of the unit cell, the second order permeability tensor is isotropic and can be depicted by a scalar $k$. The normalized permeability $k /\left(4 b^{2}\right)$ is plotted in figure 3 as a function of the ratio $a / b$ for three values of $\gamma$ (where it is recalled that $\gamma=c / a, c$ being the slip coefficient). As a validation, the FFT based solution (full line) is compared in figure 3, to a Finite Element solution (circles) for $\gamma=0$. The FEM solution has been obtained by using the COMSOL software, the computation being performed with 3584 triangular elements. The porosity is given by $\phi=1-\pi a^{2} /\left(4 b^{2}\right)$. The maximum theoretically achievable volume fraction of the cylinders is $\pi / 4 \simeq 0.785$, when $a=b$, the porosity is then given comprised between $\phi=0.215$ and $\phi=1$. It can be observed that the permeability takes the same value for $a=0$ and $a=b$, whatever the value of 
$\gamma$. In the case $a=0$ there are no inclusions whereas in the case $a / b=1$ no flow can occurs. However, for intermediate values of $a / b$, the permeability increases with $\gamma$. On figure 4 is plotted the normalized permeability $\bar{k}(\gamma=1)=k(\gamma=$ $1) / k(\gamma=0)$ as a function of the ratio $a / b$. It can be observed that $\bar{k}(\gamma=1)$ increases with the radius of the cylinders, showing that the permeability is strongly affected by the slip on the boundary when the cylinders are closely spaced.

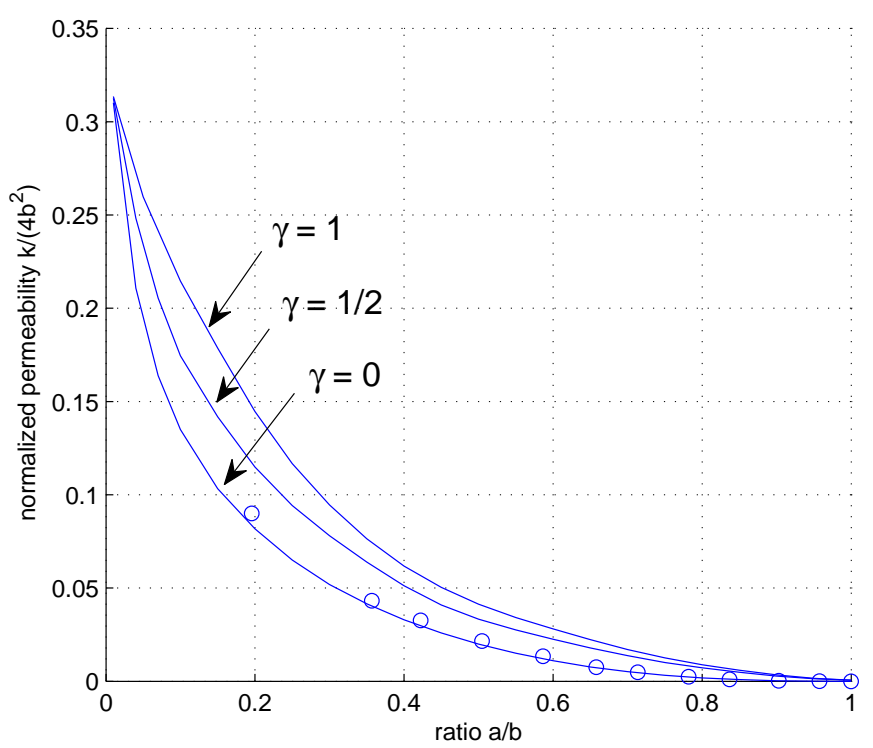

Figure 3. Normalized permeability $k /\left(4 b^{2}\right)$ as function of the ratio $a / b$.

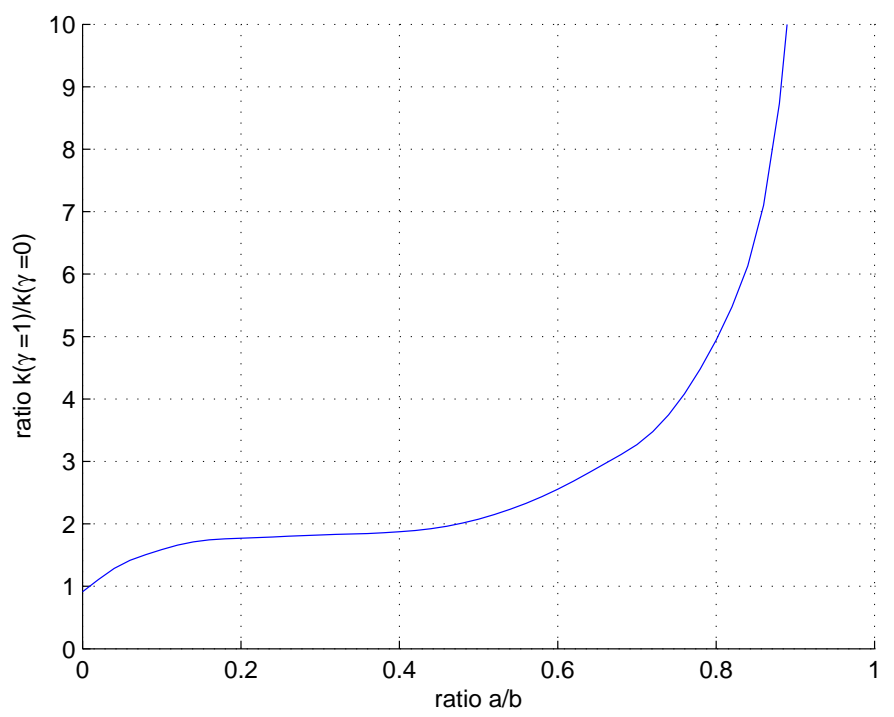

Figure 4. Ratio $k(\gamma=1) / k(\gamma=0)$ as function of the ratio $a / b$. 


\section{Conclusion}

A FFT based-method for computing the permeability of a periodic microporous media saturated by a viscous fluid has been proposed in the present paper. The components of the permeability are obtained by determining the velocity field over the unit cell due to a prescribed macroscopic gradient pressure. The slip effect on the permeability, such as in the case of rarefied gases or micropores, is also studied in this paper. The methodology used to introduce this slip condition is to consider an interphase between the fluid and the solid whose thickness tends to zero. As an illustration purpose, the method is applied for the computation of the permeability of a porous medium made up of aligned rigid cylinders. The results show that the permeability increases with the slip coefficient. It has been also shown that the effect of the slip condition increases with the cylinders radius and is maximal when the inclusions are closely spaced. The method for taking into account interface effects described in this paper can be obviously translated to the case of elasticity for studying nano-composites.

\section{References}

[1] A.S. Sangani, A. Acrivos. Slow flow past periodic arrays of cylinders with application to heat transfer. Int. J. Multiphase Flow. Vol. 8, pp. 193-206, 1982.

[2] J. Barrère, JP Caltagirone , O. Gipouloux. Détermination numérique de la perméabilité en milieu poreux périodique tridimensionnel. C.R. Acad. Sc. Paris Série II. Vol. 310, pp. 347-352, 1990.

[3] C.Y. Wang. Stokes flow through a rectangular array of circular cylinders. Fluid Dynamics Research. 29, pp. 65-80, 2001.

[4] A. Kadaksham, S.B. Pillapakkam, P. Singh. Permeability of periodic arrays of spheres. Mechanics Research Communications. Vol. 32, pp. 659-665, 2007.

[5] C.Y. Wang. Stokes slip flow through square and triangular arrays of circular cylinders. Fluid Dynamics Research. Vol. 32, pp. 233-246, 2003.

[6] H. Moulinec, P. Suquet. A fast numerical method for computing the linear and nonlinear properties of composites. C.R. Acad. Sc. Paris Série II. Vol. 318, pp. 1417-1423, 1994.

[7] W. Brown. Solid mixture permittivities. J. Comput. Math. Vol. 23, 1514-1517, 1955.

[8] E. Kroner. Statistical Continuum Mechanics (Springer-Verlag, Wien, 1972).

[9] J.C. Michel, H. Moulinec, P. Suquet. A computational scheme for linear and non-linear composites with arbitrary phase contrast. Int. J. Numer. Meth. Engng. Vol.52 pp. 139-160, 2001. 
[10] G. Bonnet. Effective properties of elastic periodic composite media with fibers. J. Mech. Phys. Solids. Vol. 55, pp. 881-899, 2007.

[11] Y. Benveniste, T. Miloh. Imperfect soft and stiff interfaces in twodimensional elasticity. Mechanics of Materials. Vol. 33, Issue 6, pp. 309-323, 2001.

[12] H.I. Ene, E. Sanchez-Palencia. Equations and surface phenomena for the flow in a porous medium. J. Mécanique. Vol. 14-1, pp. 73-108, 1975.

[13] J.L. Auriault, E. Sanchez-Palencia. Study of macroscopic behavior of a deformable porous medium. J. Mécanique. Vol. 16-4, pp.575-603, 1977.

[14] T. Levy. Fluid flow through an array of fixed particles. Int. J. Eng. Sci. Vol. 21-1, pp. 11-23, 1983.

[15] E. Sanchez-Palencia. Nonhomogeneous Media and Vibration Theory, Lecture Notes in Physics, vol. 127, 1980. Springer, Berlin.

[16] G. Allaire. Homogenization of the Navier-Stokes equations with a slip boundary condition. Communications on Pure and Applied Mathematics. Vol. 44, pp. 605-641, 1991.

[17] D. Cioranescu, P. Donato. Homogenization of the Stokes problem with nonhomogeneous slip boundary condition. Mathematical Methods in the Applied Sciences. Vol. 19, pp. 857-881, 1996. 